NEWS

\title{
Cognitive behavioral therapy may be only mildly effective for anxious, autistic children
}

\section{BY NIKO MCCARTY}

16 NOVEMBER 2021

\section{Listen to this story: \\ https://www.spectrumnews.org/wp-content/uploads/2021/11/audio- a7092ba9-dd2e-4594-8281-2f552919c624-encodings.mp3}

Autistic children tend to rate cognitive behavioral therapy (CBT) as less effective at treating their anxiety than their parents and clinicians do, according to a meta-analysis of 19 randomized clinical trials.

The result, based on data from 833 autistic children up to 18 years old, raises concerns about how clinical trials measure anxiety in young people, and who benefits from CBT.

"We fundamentally need to ask ourselves: If it's working, then whom is it working for?" says study lead Shivani Sharma, head of the psychology division at the University of Hertfordshire in the United Kingdom. "Because it's really odd that there's such a difference between the clinician ratings, the parent ratings, and then the person themselves, their own rating."

!function()\{"use strict";window.addEventListener("message",(function(e)\{if(void $0 !==$.data["datawrapper-height"])\{var $\mathrm{t=document.querySelectorAll("iframe");for(var} \mathrm{a} \mathrm{in}$ e.data["datawrapper-height"])for(var $r=0 ; r$ 